\title{
Berufungsakten als Datensorte für die Geschichte der Soziologie
}

Julian Hamann, Forum Internationale Wissenschaft, University of Bonn

\begin{abstract}
Der Beitrag stellt Berufungsakten als empirisches Material für die Soziologiegeschichte vor. Berufungsakten gewähren Einblicke in formelle und offizielle Praktiken der Selbst- und Fremdpositionierung von Wissenschaftlern. In den Dokumenten dieser reglementierten Verfahren der Berufung kommen unterschiedliche Zuschreibungen und Kategorisierungen zum Ausdruck, die andere empirische Zugriffe in dieser Form nicht erfassen können. Nach einem Überblick über den Forschungsstand zu Berufungsverfahren im Allgemeinen werden im Hauptteil des Beitrags archivrechtliche Fragen des Zugangs zu und des Inhalts von Berufungsakten geklärt. Darauf folgen Überlegungen zu den methodologischen Potenzialen dieser Dokumente. Die im Beitrag vertretene These lautet, dass die in Berufungsakten dokumentierten formellen Praktiken der Positionierung eine eigene, ebenso wirkmächtige und soziologisch relevante Realität der Verfahren abbilden wie die informellen Praktiken, die in den Akten nicht enthalten sind.
\end{abstract}

\section{Einleitung}

Wissenschaft ist ein soziales Unterfangen, das nicht nur auf die Produktion von Erkenntnis ausgerichtet ist (für konstruktive Hinweise zu früheren Versionen dieses Textes danke ich Mila Brill und Daniela Ruß.). ${ }^{1}$ Darüber gelegt ist ein Netz von feinen Differenzen und Kategorisierungen. Dies ist das diskursive Arsenal, aus dem sich Wissenschaftler über Subjektpositionen in vertikale und horizontale Ordnungen bringen. Solche Positionierungen mögen besonders pointiert und folgenreich in Berufungsverfahren stattfinden, sie sind aber keineswegs darauf beschränkt. Das von Wissenschaftlerinnen situativ und praktisch beherrschte Spiel feiner Unterscheidungen ist elementarer Teil des akademischen Alltags. Die dabei mobilisierten Kategorien und Zuschreibungen lassen sich zumindest analytisch in drei Typen trennen (vgl. Angermuller 2013, 2016): Für Selbst- und Fremdpositionierungen sind, erstens, akademische Kategorien inhaltlich-fachlicher Art zentral. Wozu arbeitet zum Beispiel die Person, die ich auf der letzten Konferenz kennengelernt habe? Wer vertritt meinen Forschungsbereich im neu erschienenen Handbuch? Zweitens sind auch institutionelle Kategorien bürokratisch-organisationaler Art wichtige Elemente von Selbstund Fremdpositionierungen. Übernimmt der Juniorprofessor an meinem Institut die gleichen Verwaltungsaufgaben wie die W3-Professorin? Wer wird in den Vorstand der Deutschen Gesellschaft für Soziologie gewählt? Drittens spielen auch nicht-akademische Kategorien eine Rolle für die Selbst- und Fremdpositionierungen von Wissenschaftlerinnen. Sozialstrukturelle Determinanten wie soziale Herkunft, Geschlecht oder Ethnie, aber auch 
Positionen außerhalb der Wissenschaft in der Politik, den Medien oder der Wirtschaft, können zu sehr relevanten Faktoren akademischer Positionierungen werden und den Verlauf wissenschaftlicher Karrieren beeinflussen.

In diesen ernsten Spielen der feinen Unterscheidungen und Kategorisierungen können sich Wissenschaftlerinnen beispielsweise vertikal in Positionen wie „Doktorand“, „Postdoc“ oder „W3-Professorin“ einordnen, oder sie verorten sich horizontal über Positionen wie „Sportsoziologin“, „Diskursforscher“ oder „Theoretikerin“. Es versteht sich von selbst, dass die Stabilität und Fixierung dieser Ordnungen stark variiert. Über die produktive, situative und kontingente Seite dieser Positionierungen informieren beispielsweise praxeologische Perspektiven (Langenhove und Harré 1999; Baert 2012), die robusten und strukturellen Aspekte dieser sozialen Ordnungen werden zum Beispiel von macht- und elitesoziologischen Perspektiven betont (Bourdieu 1992; Graf 2015).

Eine besonders instruktive Arena akademischer Positionierungen sind Berufungsverfahren. Sie bilden einen verfahrensmäßigen Rahmen für das akademische Spiel der feinen Unterscheidungen und reglementieren, auf welche Weise wissenschaftliches Leitungspersonal institutionell in Stellung gebracht wird. Berufungsverfahren übersetzen also flexible und situative Positionierungen in die robuste und stabile Ordnung institutioneller Posten und Hierarchien. So werden beispielsweise jene spontanen Fremd- und Selbstzuschreibungen, die im Gespräch nach dem Probevortrag oder in den Diskussionen der Berufungskommission stattfinden, in die dauerhafte institutionelle Kategorie der Professur überführt. Weil Berufungsverfahren akademische (und nicht-akademische) Unterscheidungen in die institutionelle Kategorie „Professur für ...“ übertragen, gibt es in der Wissenschaft kaum zentralere Arenen der Positionierung. Das Spiel der feinen Unterscheidungen ist nirgendwo ernster als hier.

Im vorliegenden Beitrag werde ich Möglichkeiten und Potenziale des soziologiegeschichtlichen Zugriffs auf Berufungsakten aufzeigen. Dazu gebe ich zunächst einen Überblick über die wichtigsten Forschungslinien zu Berufungsverfahren im Allgemeinen (2.). Dem folgt eine Diskussion forschungspraktischer Aspekte, wozu archivrechtliche Fragen des Zugangs zu und des Inhalts von Berufungsakten gehören (3.). Nach Überlegungen zu den methodologischen Potenzialen von archivierten Dokumenten (4.) können abschließend die wichtigsten Analyseperspektiven auf Berufungsakten zusammengefasst werden (5.).

\section{Forschungsstand zu Berufungsverfahren}

Das Feld akademischer Positionierungen kann prinzipiell über verschiedene empirische Daten und Gegenstände erschlossen werden. Dafür kommen Ansätze in Frage, die fast die gesamte Welt wissenschaftlicher Positionierungspraktiken spiegeln: Studien zu Positionierungen in Zitationen (z. B. Allen 1997), in Empfehlungsschreiben (z. B. Tsay et al. 2003), in Nachlässen (z. B. Füß1 2014), in (Auto-)Biografien (z. B. Platt 2015, siehe auch ihren Beitrag in diesem Band) oder in Nachrufen (z. B. Hamann 2016, siehe auch den Beitrag in diesem Band) sowie zu außerwissenschaftlichen Positionierungen in Medien und Wirtschaft (z. B. Maeße 2015) eröffnen eine vergleichende Perspektive, auf die sich die Erforschung von Selbst- und Fremdpositionierungen in Berufungsverfahren stützen kann. 
Solche Vergleiche sind nicht zuletzt hilfreich, weil Berufungsverfahren selbst bisher nur schlaglichtartig erforscht sind. Erstens konzentriert sich eine Reihe von Studien auf wissenschaftspolitische, praxisnahe Aspekte und formuliert normative Erwartungen an Berufungsverfahren. Es wird herausgearbeitet, dass Universitäten in immer stärkerem Ausmaß zu Konkurrenten auf einem Berufungsmarkt werden, und dass auch Wissenschaftler systematisch dazu angehalten werden, miteinander in einen Wettbewerb um Positionen zu treten (Detmer 2012; Färber und Riedler 2011; Wissenschaftsrat 2005). Im Ländervergleich zeigen sich die (pfadabhängigen) Strukturen nationaler Hochschulsysteme (Schmitt et al. 2004; Fumasoli und Goastellec 2015) sowie unterschiedliche Bewertungsmaßstäbe und kriterien der jeweiligen Gremien (Musselin 2005).

Neben diesen Arbeiten existiert ein zweiter Forschungsstrang zur Bedeutung von akademischen und nicht-akademischen Zuschreibungen in Berufungsverfahren. Hier zeigt sich, dass Berufungen beeinflusst werden von Forschungsleistungen (zugeschrieben durch Publikationen und Drittmitteleinwerbungen) und Lehrqualitäten (markiert durch Lehrevaluationen) (Kleimann et al. 2015; Lutter und Schröder 2014; Plümper und Schimmelpfennig 2007). Auch das Geschlecht und die sozialen Netzwerke der Bewerber haben einen Einfluss auf ihre Berufungschancen (zum Einflussfaktor Geschlecht siehe Misra et al. 1999; Färber und Spangenberg 2008; Nielsen 2016, zu Netzwerken Combes et al. 2008; Gross et al. 2008). Bereits diese kurze Skizze von sich in Berufungsverfahren überkreuzenden akademischen und nicht-akademischen Zuschreibungen deutet die Komplexität wissenschaftlicher Rekrutierungsprozesse an. Wenn Wissenschaftlerinnen institutionell in Stellung gebracht werden, sind darüber hinaus auch solche Faktoren zu berücksichtigen, die sich schwerer quantifizieren lassen. Dazu gehören die durch Selbst- und Fremdzuschreibungen konstruierte Passfähigkeit von Kandidaten (Zimmermann 2000), die Einhaltung bestimmter Spielregeln des Verfahrens (Kriszio 1994) und die durch nationale Verfahrensmodelle strukturierten Entscheidungs- und Bewertungspraktiken (Musselin 2009).

Für die Fragestellung meines Beitrags sind die Datengrundlagen der jeweiligen Studien ebenso wichtig wie die Zuschreibungen und Kategorien, die jeweils fokussiert werden. Die entscheidende Rolle des empirischen Zugriffs zeigt sich zum Beispiel beim Faktor Geschlecht: Während quantitative Längsschnittanalysen von Lebenslaufdaten weder für die Politikwissenschaft (Plümper und Schimmelpfennig 2007) noch für die Soziologie (Mau und Huska 2010; Lutter und Schröder 2014) eine systematische Benachteiligung von Frauen nachweisen (anders sieht das bei Müttern aus), können qualitative Studien, die auf teilnehmender Beobachtung und Experteninterviews beruhen, differenziertere Aussagen treffen. Sie unterscheiden nicht nur, ob Frauen entweder berufen oder ausgegrenzt werden, sondern verweisen darauf, wie Vorstellungen von Männlichkeit Rekrutierungspraktiken in den Gremien und Kommissionen prägen (Zimmermann 2000; Färber und Spangenberg 2008).

Als Zwischenfazit können wir festhalten, dass die bisher vorgestellten Studien Berufungsverfahren unter Rückgriff auf unterschiedliches empirisches Material untersuchen. Schematisch lassen sich drei Zugangsweisen unterscheiden. Erstens können wissenschaftsund hochschulpolitische Gesetze, Regeln und Leitlinien konsultiert werden, aus denen sich Ziele und Forderungen ableiten lassen, die aber auch internationale Vergleiche informieren können. Zweitens können Berufungsverfahren anhand von Lebenslaufdaten Berufener untersucht werden. Hier handelt es sich gewissermaßen um Analysen von außen, die die Ergebnisse von Verfahren ex post anhand der Sozialstruktur der Professorenschaft erfassen. Der Vorteil dieses Zugangs besteht darin, dass die Merkmale erfolgreicher Kandidatinnen relativ detailliert und über größere Zeiträume hinweg studiert werden können. Einen 
differenzierten Blick in die Black Box ermöglicht, drittens, die teilnehmende Beobachtung von Berufungsverfahren. Sie kann um Experteninterviews ergänzt werden. Beide Zugangsweisen bergen jedoch das Risiko, dass Forscherinnen durch ihre bloße Anwesenheit oder ihr Frageverhalten einen Einfluss auf die Situation ausüben, der nur schwer zu kontrollieren ist.

Auch die Wissenschaftsgeschichte hat Berufungsverfahren bisher nur am Rande thematisiert. In der Regel werden dabei Fachgeschichten oder Universitätsgeschichten geschrieben; bei Ersteren bildet die Historiografiegeschichte einen Schwerpunkt (Hesse und Schwinges 2012; Dainat 2003). Typischerweise fokussieren auch diese Studien den Einfluss nichtakademischer Faktoren wie Geschlecht und die Zugehörigkeit zu Schulen, oder sie zeichnen (Dis-)Kontinuitäten in der Berufungspolitik von Instituten und Universitäten nach. Untersuchungen können dabei Professorenlexika und -kataloge (Asche 2012; Morgenstern 2012), Interviews (Stambolis 2012) oder Briefwechsel und Korrespondenzen heranziehen (Rebenich 2012). Wie in Analysen der aktuellen Sozialstruktur gibt es auch in der Wissenschaftsgeschichte quantitative Arbeiten zum Berufungswandel, die sich auf Lebenslaufdaten stützen. Ergebnisse von Berufungsverfahren gewähren Einsichten in die Persistenz wissenschaftlicher Schulen (Weber 1984), die Differenzierung von Geistes- und Sozialwissenschaften (Baumgarten 1997) und geschlechtsspezifische Diskriminierung (Paletschek 2012). Auch diese Untersuchungen konzentrieren sich aber auf die ex postEffekte von Berufungen und bleiben außerhalb der Verfahren selbst. Berufungsakten werden entweder ergänzend in Einzelfallstudien oder in wenigen universitäts- und institutsgeschichtlichen Arbeiten herangezogen (z. B. Hofmann 1982).

In den folgenden Abschnitten will ich näher auf Berufungsakten als Datenmaterial eingehen. Dazu diskutiere ich einige forschungspraktische und methodologische Aspekte, die bei der Arbeit mit Berufungsakten im Rahmen der Soziologiegeschichte relevant sind. Die Einblicke sind in einem DFG-Projekt (Laufzeit 2015-2018) an der Universität Bonn entstanden, in dem ich Berufungsverfahren in disziplinären Umbrüchen in Geschichtswissenschaft und Germanistik in den 1960er- und 1970er-Jahren erforsche.

\section{Forschungspraxis: Zugang zu Berufungsakten und in den Akten enthaltene Dokumente}

Zunächst stellt sich die archivrechtliche Frage des Zugangs zu Berufungsakten. Die rechtlichen Rahmenbedingungen der Einsichtnahme sind geregelt durch

Landesarchivgesetze, die dann von den jeweiligen Universitätsarchiven ausgelegt werden. Nach dieser Auslegung sind Berufungsakten nicht als Sachakten, sondern als personenbezogene Akten definiert. Dadurch wird nach allen Landesarchivgesetzen eine gegenüber Sachakten verlängerte Sperrfrist notwendig. Prinzipiell kann eine Einsicht dennoch unter drei Bedingungen gewährt werden, die zwischen den Bundesländern nur minimal variieren:

- Die personenbezogene Sperrfrist ist beendet, wenn die Verfahrensbeteiligten seit mindestens zehn Jahren verstorben sind. Wer zum Kreis der Verfahrensbeteiligten gehört, wird in der Praxis unterschiedlich ausgelegt. Ob hierunter nur berufene Personen oder auch andere gelistete Kandidatinnen, Kommissionsmitglieder oder Gutachterinnen fallen, liegt in der Entscheidung der jeweiligen Universitätsarchive. 
- Die personenbezogene Sperrfrist wird temporär aufgehoben, wenn die Verfahrensbeteiligten ihr Einverständnis in die Akteneinsicht geben. Falls ihr Tod weniger als zehn Jahre zurückliegt, kann dieses Einverständnis auch von Angehörigen gegeben werden.

- Die personenbezogene Sperrfrist kann verkürzt werden, wenn dies für wissenschaftliche Forschungsvorhaben beantragt wird. Hier müssen besondere wissenschaftliche Interessen in einem Antrag dargelegt werden.

Wie auch bei anderen Archivdaten (vgl. nur Fabian Links Beitrag zu Korrespondenzen in diesem Band) werden die Fragen des Zugangs zu Berufungsakten von Universitätsarchiven entschieden. Bereits die Bedingungen für Sperrfristverkürzungen und -aufhebungen zeigen, dass dabei zwei widerstreitende Interessen vereinbart werden müssen: wissenschaftliche Forschungsinteressen und die schutzbedürftigen Interessen der Verfahrensbeteiligten (Hoen 2010). Die schutzbedürftigen Interessen der Beteiligten leiten sich aus dem Recht auf informationelle Selbstbestimmung ab. Das Selbstbestimmungsrecht steht aber in Konflikt mit der grundgesetzlich zugesicherten Forschungsfreiheit. Auch personenbezogene Informationen sind Teil einer forschungsrelevanten sozialen Realität (Manegold 2013). Die Gewichtung dieser beiden Interessen nehmen Universitätsarchive je unterschiedlich vor, so dass auch die Möglichkeiten der Einsichtnahme variieren.

In der Regel ist die Einsichtnahme mit besonderen Auflagen verbunden. Grundsätzlich sind die schützenswerten Belange der Betroffenen zu berücksichtigen. Dies kann durch eine Anonymisierung relevanter Informationen erreicht werden. Üblicherweise dürfen Informationen nur im Rahmen eines konkreten Forschungsprojekts verwendet werden; nicht immer ist es erlaubt, von den Akten Reproduktionen anzufertigen. Vervielfältigte Unterlagen und auch transkribierte Informationen sind grundsätzlich so aufzubewahren und zu speichern, dass unbefugte Personen keinen Zugriff haben.

Weil die verschiedenen Schritte eines Berufungsverfahrens auf unterschiedlichen Ebenen archiviert werden, sind die Fragen des Zugangs und der Einsichtnahme je nach Fragestellung mit verschiedenen Stellen zu klären. Dokumente zu einem einzigen Verfahren können über Ministerialakten, Rektorats- und Senatsakten, Fakultätsakten und Akten der Berufungskommission verstreut sein. Ungeachtet der Kompetenzen der Universitätsleitungen und Ministerien dürften die Berufungsakten der Fakultäten und der Berufungskommissionen für die meisten soziologiegeschichtlichen Fragestellungen das interessanteste Datenmaterial darstellen. In den hier dokumentierten Verfahrensschritten schlagen sich Fremd- und Selbstpositionierungen der Beteiligten besonders deutlich nieder, weil in diesen Schritten die Passung zwischen Bewerber- und Stellenprofil vorgenommen wird. Die in der Regel im Universitätsarchiv des Berufungsorts archivierten Akten umfassen je nach Vollständigkeit unter anderem Ausschreibungstexte, Bewerbungsschreiben inklusive Curricula Vitae, Schriften- und Lehrverzeichnissen, Protokolle der Kommissionssitzungen, Briefwechsel und Korrespondenzen, externe Gutachten und Laudationes über die Listenplatzierten sowie den Verlauf und die Ergebnisse der Berufungsverhandlungen. Ob und in welchem Umfang eine Forschungsfrage bearbeitet werden kann, hängt demnach nicht nur von der Möglichkeit des Zugangs zu den Akten ab, sondern auch von der im Vorfeld noch schwerer abzuschätzenden Vollständigkeit der Akten. Nicht selten ist es notwendig, Datenmaterial und/oder Forschungsfrage im Nachhinein neu auszutarieren und aneinander anzupassen (dazu Elder et al. 1993). 


\section{Methodologische Potenziale von Berufungsakten}

Ebenso wichtig wie Fragen des archivrechtlichen Zugangs und der Inhalte von Berufungsakten ist die Diskussion ihres methodologischen Stellenwerts. Hierzu können wir kurz auf die im Forschungsstand angesprochenen Stärken und Schwächen anderer empirischer Zugänge zurückkommen. Ich hatte oben angemerkt, dass teilnehmende Beobachtung und Experteninterviews zwar einen vergleichsweise differenzierten Blick in die Black Box erlauben. Die Reaktivität der Daten stellt jedoch eine Schwäche dieser Erhebungstechniken dar. Forscherinnen können durch ihr Frageverhalten oder durch ihre bloße Anwesenheit auf die erhobenen Daten einwirken. Im heiklen Kontext von Personalbewertungen und -entscheidungen ist diese Einschränkung besonders wahrscheinlich, weil es nicht um Projektanträge oder Manuskripte, sondern um Kollegen und Konkurrentinnen geht. Die aktenmäßige Dokumentation von Berufungsverfahren (und weiteren soziologiegeschichtlich relevanten Prozessen) unterliegt anderen Beschränkungen, sie eröffnet aber auch andere Potenziale.

Dokumente im Allgemeinen und Akten im Besonderen können zum einen historiografischpositivistisch als „Quelle“ und zum anderen diskursanalytisch-ethnomethodologisch als „Phänomen“ verstanden werden (Zimmerman und Pollner 1971; Prior 2008, 2012). Der ersten Perspektive geht es um Belege und Beweise, die Dokumenten als stummen, reglosen, nicht-reaktiven Quellen entnommen werden (May 2001, S. 175-199; Bryman 2012, S. 542563). Berufungsakten geben dann Auskunft über den Wortlaut von Stellenausschreibungen, über erfolgreiche Kandidaten und den Kreis der Mitbewerberinnen, über Listenplatzierungen, Gutachter oder die Dauer der Verfahren. Viele darüber hinausgehende Informationen müssen jedoch aus quellenkritischer Perspektive hinterfragt werden. Es ist schlichtweg nicht aktenkundig, welche Vorbehalte Kommissionsmitglieder gegen Kandidatinnen geltend machen, ob die enthaltenen Gutachten bereits mit Blick auf eine präferierte Entscheidung ,bestellt“" wurden, ob die Kommissionsprotokolle vom Ergebnis her geschrieben und Abstimmungen so oft vorgenommen wurden, bis das Abstimmungsergebnis stimmte. Die positivistische Perspektive auf Dokumente und Akten kann Berufungsakten aber trotz dieser Einschränkungen durchaus gewinnbringend als Datenmaterial verwenden, wenn sie ihre Aussagekraft nicht an entscheidenden Punkten überschätzt.

Alternativ können Dokumente diskursanalytisch-ethnomethodologisch als eigenes und entsprechend selbst zu beforschendes „Phänomen“ begriffen werden - auch wenn die Unterscheidung zwischen „Quelle“ und „Phänomen“ eine analytische, in der Praxis nicht immer klar zu treffende ist. In der Wissens- und Wissenschaftssoziologie sind jedenfalls schon in ganz ähnlicher Weise Statistiken untersucht worden. Auch hier steht dann nicht mehr die Evidenz von Quellen, sondern ihre Performativität im Mittelpunkt (siehe nur Desrosières 1998; Heintz 2012; Mair et al. 2016). Für archivierte Dokumente bedeutet diese Perspektivenverschiebung, dass Akten nicht länger als passive Container für Inhalte, sondern als Agenten betrachtet werden, die mit ihrer Umwelt interagieren und konkrete Funktionen haben (Cooren 2004; vgl. auch Latour 1988). Der Fokus verschiebt sich also von der Frage, was Berufungsakten belegen und zeigen, zu der Frage, wie sie in organisatorischen, bürokratischen oder akademischen Kontexten produziert werden, wie sie in diesen Kontexten zirkulieren, wie sie verwendet werden, und wie Akten selbst auf ihre verschiedenen Kontexte zurückwirken (vgl. Trace 2002; Smith 2007; Atkinson und Coffey 2004). Diese diskursanalytisch-ethnomethodologische Perspektive auf Berufungsakten bewegt sich nicht weg von den Inhalten von Gutachten, Lebensläufen oder Protokollen. Sie beleuchtet vielmehr, wie diese Elemente funktionieren und wirken, wie sie produziert, rezipiert, genutzt und manipuliert werden. Auch wenn diese Aspekte einer auf Quellen konzentrierten 
historiografisch-positivistischen Sicht verschlossen bleiben müssen, gibt es in der Wissenschaftsgeschichte ähnliche Bestrebungen, wissenschaftliche Objekte von ihren Entdeckern oder Benutzern zu emanzipieren (Daston 1999).

Inwiefern verändert sich also unser analytischer Blick, wenn wir Berufungsakten nicht als „Quellen“ verstehen, die die Realität dann immer nur unzureichend wiedergeben können, sondern als „Phänomen“ begreifen? Schematisch können informelle Praktiken der Positionierung und Unterscheidung in Berufungsverfahren angenommen werden, die nicht völlig deckungsgleich mit den formellen Praktiken sind, die sich dann aktenmäßig niederschlagen. Informell können hinter verschlossener Tür oder in der Kaffeepause Zuschreibungen getätigt werden, die öffentlich nicht legitim - oder zumindest: nicht legitim wirkmächtig - wären, zum Beispiel „Arbeiterkind“ oder „Migrantin“. Es können Gefallen erwiesen und ein Konsens gefunden, aber auch der Dissens zelebriert und Privatfehden ausgetragen werden. Es können individuelle Erwägungen einfließen, beispielsweise Sympathie, aber auch Konkurrenzdenken. Natürlich können in Berufungsverfahren auch Zuschreibungen akademischer Leistungsfähigkeit vorgenommen und zur primären Entscheidungsgrundlage gemacht werden. Der Punkt ist nicht, ob diese informellen Praktiken durchweg aktenkundig werden (sie werden es nicht), ob sie immer anerkannt und legitim sind (sie sind es nicht), oder ob die vorgenommenen Zuschreibungen von anderen Erhebungsmethoden wie teilnehmender Beobachtung grundsätzlich besser erfasst werden (sie werden es nicht). Insofern würde eine Kritik ins Leere laufen, die bemängelte, dass Berufungsakten nicht „die Realität“ der Verfahren erfassen. Die in Berufungsakten dokumentierten formellen Praktiken bilden eine eigene, ebenso wirkmächtige und soziologisch relevante Realität der Verfahren ab wie die informellen Praktiken, die in den Akten nicht immer auftauchen.

Vermeintliche Defizite des Datenmaterials werden auf diese Weise zu eigenständigen soziologischen Phänomenen und sie verweisen auf die organisatorischen, bürokratischen und akademischen Kontexte, in denen und für die Berufungsakten produziert und rezipiert werden. Mit Garfinkel (1967) gesprochen gibt es also „gute“ Gründe für „schlechte“ Akten, und bei Berufungsakten sind dies nicht zuletzt strategische Gründe: Die Akten sollen den Weg zu einer legitimen Entscheidung dokumentieren, sie sollen für Transparenz sorgen und zwischen verschiedenen Verfahrensschritten, unterschiedlichen Graden der Öffentlichkeit und heterogen zusammengesetzten Kommissionen Kohärenz herstellen. Nicht zuletzt sollen Berufungsakten verhindern, dass getroffene Entscheidungen im Nachhinein anfechtbar sind (Stark 2011). Angesichts dieser vielfältigen strategischen Zwecke können wir davon ausgehen, dass die objektive Dokumentation des ,tatsächlichen“"Verfahrensablaufs in Berufungsakten nicht die erste Priorität der Verfahrensbeteiligten ist. Dies ist weder ein pauschales Argument gegen historiografisch-positivistische Verwendungsweisen von Berufungsakten als „Quelle“ noch ein Argument für ihre diskursanalytischethnomethodologische Untersuchung als „Phänomen“. Zunächst heißt das nur, dass die Passung zwischen Forschungsfrage und methodologischer Perspektive bei Berufungsakten besonders sorgfältig reflektiert werden muss.

\section{Fazit}

Auf die oben diskutierten methodologischen Potenziale aufbauend fasse ich zusammen, welche unterschiedlichen Analyseperspektiven Berufungsakten eröffnen. Ich skizziere dazu idealtypisch vier Perspektiven, die sich in der Forschungspraxis ergänzen und überschneiden 
können. Dabei lehne ich mich an Priors (2008, S. 825) tabellarische Unterscheidung von Approaches to the study of documents an (Tab. 1).

Tab. 1: Analytische Perspektiven auf Berufungsakten (Tabelle in Anlehnung an Prior (2008, S. 825))

\begin{tabular}{l|l|l} 
& Berufungsakten als Quelle & Berufungsakten als Phänomen \\
\hline Inhalt & $\begin{array}{l}\text { 1) Was wird durch den Inhalt der } \\
\text { Dokumente ,belegt“? }\end{array}$ & $\begin{array}{l}\text { 3) Wie entstehen die Inhalte der } \\
\text { Dokumente? }\end{array}$ \\
\hline $\begin{array}{l}\text { Verwendungsweise } \\
\text { und Funktion }\end{array}$ & $\begin{array}{l}\text { 2) Wie werden Dokumente } \\
\text { strategisch verwendet? }\end{array}$ & $\begin{array}{l}\text { 4) Wie wirken Dokumente in } \\
\text { sozialer Ordnung und Interaktion } \\
\text { und beeinflussen diese? }\end{array}$
\end{tabular}

Werden Berufungsakten positivistisch ausschließlich als Quellenmaterial verstanden, kann sich eine Analyse, erstens, auf die bürokratische Dimension der Verfahren konzentrieren. Akten geben dann Auskunft über die Dauer von Berufungsverfahren, über den Ablauf einzelner Verfahrensschritte oder über die Personen(gruppen), die offiziell am Verfahren beteiligt sind. Im Sinne von Quellen belegen Akten auch, welche Argumente offiziell verwendet werden können, um Personalentscheidungen zu begründen. Die historiografischpositivistische Perspektive kann auch, zweitens, die strategische Verwendung der Akten thematisieren. So sind Berufungsakten ein zentrales Mittel, um den Ablauf eines Verfahrens transparent zu machen. Protokolle halten dann zum Beispiel fest, wer an Kommissionssitzungen beteiligt war und ob die Kommission paritätisch zusammengesetzt war. Eine ähnliche Funktion haben Kommissionsberichte, die einen schnellen Überblick über das Verfahren gewähren. Um der strategischen Verwendung von Akten Rechnung zu tragen, müssen die enthaltenen Dokumente explizit auch intertextuell, also in ihrer verfahrensmäßigen Sequenz betrachtet werden. Die Stellenausschreibung ist beispielsweise ein wichtiger Hintergrund zur Bewertung von Bewerbungsunterlagen. Die dort hervorgebrachten Bewerberprofile können dann wiederum auf einer Berufungsliste platziert werden, deren Reihung durch Laudationes legitimiert werden soll.

Die Analyse von Berufungsakten kann, drittens, ethnomethodologisch nach dem mobilisierten Distinktionswissen fragen. Aus diesem Blickwinkel erkennen wir nicht nur, welche Argumente offiziell verwendet werden, um Entscheidungen zu begründen. Vielmehr reflektieren die Dokumente, wie die am Verfahren beteiligten Akteure ihr Wissen nutzen. Sie beziehen sich auf anerkannte Unterscheidungen und nehmen legitime Positionierungen vor. Die Akten stellen aus dieser Sicht Arenen der formalisierten Selbst- und Fremdpositionierung dar, in denen akademische und nicht-akademische sowie institutionelle Kategorien und Zuschreibungen mobilisiert werden.

Viertens sensibilisiert ein diskursanalytischer Zugriff auf Berufungsakten dafür, dass Texte nicht nur strategisch verwendet werden, sondern auch eine eigene Wirkmächtigkeit entfalten. Unterschiedliche Dokumente wie Stellenausschreibungen, Kommissionsprotokolle, Gutachten oder Laudationes wirken im Verfahren auf unterschiedliche Weise. Sie sortieren heterogene Unterscheidungen und Erwartungen innerhalb der Kommission, sie überführen individuelle Positionierungen in eine kollektive Entscheidung, sie dokumentieren und legitimieren das Verfahren oder schließen es nach außen hin ab. Es kann vor diesem Hintergrund nicht überraschen, dass sich die verschiedenen Dokumente dabei 
unterschiedlicher sprachlicher Register bedienen. Weiterhin gibt die Kommunikation zwischen aufeinanderfolgenden Dokumenten den Blick darauf frei, wie die feinen Unterscheidungen und Kategorisierungen im Verfahren zu diskursiven Subjektpositionen gerinnen („Listenplatzierter“, „Kandidatin“, „Kommissionsmitglied“, „,das Institut“, „Berufene“). In der Regel werden im Verfahren Bewerberprofile mit Stellenprofilen in Passung gebracht. So entsteht die Subjektposition „Professorin für ...“, in der sich nicht nur bürokratische und organisatorische Befugnisse, Rechte und Pflichten kristallisieren, sondern über Denomination und Lehrstuhlstruktur auch inhaltliche Zuständigkeitsbereiche und Kompetenzbehauptungen spiegeln. In dieser Perspektive wird deutlich, dass Berufungsakten selbst einen wesentlichen Beitrag zur Konstruktion und Verfestigung akademischer Subjektpositionen leisten.

\section{Fußnoten}

1. Der Text wechselt zwischen generischem Maskulinum und Femininum. Davon unbenommen bleibt, dass Geschlecht in Berufungsverfahren eine einflussreiche Variable ist.

\section{Literatur}

Allen, Bryce. 1997. Referring to schools of thought: An example of symbolic citations. Social Studies of Science 27(6): 937-949.

Angermuller, Johannes. 2013. How to become an academic philosopher. Academic discourse as multileveled positioning practice. Sociología histórica 2013(2): 263-289.

Angermuller, Johannes. 2016. Akademische Subjektivierung. Was Statuskategorien über wissenschaftliche Karrieren in Frankreich im Vergleich zu den USA, Großbritannien und Deutschland aussagen. In Macht in Wissenschaft und Gesellschaft. Diskurs- und feldanalytische Perspektiven, Hrsg. Julian von Hamann, Jens Maeße, Vincent Gengnagel und Alexander Hirschfeld, 25-54. Wiesbaden: Springer.

Asche, Matthias. 2012. Biographische Profile und Rekrutierungsmechanismen von Professoren an kleinen und mittelgrossen protestantischen Universitäten im Heiligen Römischen Reich 16501800. In Professorinnen und Professoren gewinnen. Zur Geschichte des Berufungswesens an den Universitäten Mitteleuropas, Hrsg. Christian von Hesse und Rainer Christoph Schwinges, 185-245. Basel: Schwabe.

Atkinson, Paul, und Amanda Coffey. 2004. Analysing documentary realities. In Qualitative research. Theory, method and ractice, Hrsg. David von Silverman, 56-75. London: Sage.

Baert, Patrick. 2012. Positioning theory and intellectual interventions. Journal for the Theory of Social Behaviour 42(3): 304-324.

Baumgarten, Marita. 1997. Professoren und Universitäten im 19. Jahrhundert: zur Sozialgeschichte deutscher Geistes- und Naturwissenschaftler. Göttingen: Vandenhoeck \& Ruprecht.

Bourdieu, Pierre. 1992. Homo academicus. Frankfurt a. M.: Suhrkamp.

Bryman, Alan. 2012. Social research methods. Oxford: Oxford University Press.

Combes, Pierre-Philippe, Laurent Linnemer, und Michael Visser. 2008. Publish or peer-rich? The role of skills and networks in hiring economics professors. Labour Economics 15(2008): 423 441.

Cooren, François. 2004. Textual agency: How texts do things in organizational settings. Organization 11(3): 373-393.

Dainat, Holger. 2003. Zur Berufungspolitik in der Neueren deutschen Literaturwissenschaft 19331945. In Literaturwissenschaft und Nationalsozialismus, Hrsg. Holger von Dainat und Lutz Danneberg. Berlin: De Gruyter.

Daston, Lorraine, Hrsg. 1999. Biographies of scientific objects. Chicago: University of Chicago Press. 
Desrosières, Alain. 1998. The politics of large numbers: A history of statistical reasoning. Cambridge, MA: Harvard University Press.

Detmer, Hubert. 2012. Berufungs- und Bleibeverhandlungspraxis - normative und faktische Entwicklungstendenzen von 1990 bis 2010. In Professorinnen und Professoren gewinnen. Zur Geschichte des Berufungswesens an den Universitäten Mitteleuropas, Hrsg. Christian von Hesse und Rainer Christoph Schwinges, 127-141. Basel: Schwabe.

Elder, Glen H., Eliza K. Pavalko, und Elizabeth Colerick Clipp. 1993. Working with archival data: Studying lives. London: Sage.

Färber, Christine, und Ute Riedler. 2011. Black Box Berufung: Strategien auf dem Weg zur Professur. Frankfurt a. M.: Campus.

Färber, Christine, und Ulrike Spangenberg. 2008. Wie werden Professuren besetzt? Chancengleichheit in Berufungsverfahren. Frankfurt a. M./New York: Campus.

Fumasoli, Tatiana, und Gaële Goastellec. 2015. Global models, disciplinary and local patterns in academic recruitment processes. In Academic work and careers in Europe: Trends, challenges, perspectives, Hrsg. Tatiana von Fumasoli, Gaële Goastellec und Barbara M. Kehm, 69-93. Dordrecht: Springer.

Füß1, Wilhelm. 2014. Übrig bleibt, was übrig bleiben soll. Zur Konstruktion von Biografien durch Nachlässe. Berichte zur Wissenschaftsgeschichte 37(2014): 240-262.

Garfinkel, Harold. 1967. ,Good' organizational reasons for ,bad' clinic records. In Studies in ethnomethodology, Hrsg. Harold von Garfinkel, 186-207. Englewood Cliffs: Prentice Hall.

Graf, Angela. 2015. Die Wissenschaftselite Deutschlands. Sozialprofil und Werdegänge zwischen 1945 und 2013. Frankfurt a. M.: Campus.

Gross, Christiane, Monika Jungbauer-Gans, und Peter Kriwy. 2008. Die Bedeutung meritokratischer und sozialer Kriterien für wissenschaftliche Karrieren - Ergebnisse von Expertengesprächen in ausgewählten Disziplinen. Beiträge zur Hochschulforschung 30(4): 8-32.

Hamann, Julian. 2016. ,Let us salute one of our kind'. How academic obituaries consecrate research biographies. Poetics 56(2016): 1-14.

Heintz, Bettina. 2012. Welterzeugung durch Zahlen. Modelle politischer Differenzierung in internationalen Statistiken, 1948-2010. Soziale Systeme 18(2012): 7-39.

Hesse, Christian, und Rainer Christoph Schwinges, Hrsg. 2012. Professorinnen und Professoren gewinnen. Zur Geschichte des Berufungswesens an den Universitäten Mitteleuropas. Basel: Schwabe Verlag.

Hoen, Barbara. 2010. Im Widerstreit der Interessen. Möglichkeiten und Grenzen des Zugangs zu Archivgut. In forum: universitätsarchiv Heft 1: ,1933 - Hochschularchive und die Erforschung des Nationalsozialismus, Hrsg. Andreas von Freitäger, 57-74. Köln: Universitätsarchiv Köln.

Hofmann, Norbert. 1982. Die Artistenfakultät an der Universität Tübingen, 1534-1601. Tübingen: Mohr Siebeck.

Kleimann, Bernd, Susanne in der Smitten, und Maren Klawitter. 2015. Forschungserfahrung als Berufungskriterium. Anforderungen an zukünftige Professorinnen und Professoren. Forschung \& Lehre 2015(8): 644-645.

Kriszio, Marianne. 1994. Mechanismen akademischer Selbstrekrutierung - zu den Spielregeln von Berufungsverfahren. Hochschule ost 3(4): 9-27.

Latour, Bruno. 1988. The pasteurization of France. Cambridge: Harvard University Press.

Lutter, Mark, und Martin Schröder. 2014. Who becomes a tenured professor, and why? Panel data evidence from German sociology, 1980-2013. Research Policy 45(5): 999-1013.

Maeße, Jens. 2015. Economic experts. A discursive political economy of economics. Journal of Multicultural Discourses 10(3): 279-305.

Mair, Michael, Christian Greiffenhagen, und William Sharrock. 2016. Statistical practice: Putting society on display. Theory Culture \& Society 33(3): 51-77.

Manegold, Bartholomäus. 2013. Archivrecht. Die Archivierungspflicht öffentlicher Stellen und das Archivzugangsrecht des historischen Forschers im Licht der Forschungsfreiheitsverbürgung des Art. 5 Abs. 3 GG. Berlin: Duncker \& Humblot.

Mau, Steffen, und Denis Huska. 2010. Who is Who? Die Sozialstruktur der SoziologieProfessorenschaft in Deutschland. Kölner Zeitschrift für Soziologie und Sozialpsychologie 62(4): 751-766. 
May, Tim. 2001. Social research. Issues, methods and process. Buckingham/Philadelphia: Open University Press.

Misra, Joya, Ivy Kennelly, und Marina Karides. 1999. Employment chances in the academic job market in sociology: Do race and gender matter? Sociological Perspectives 42(2): 215-247.

Morgenstern, Ulf. 2012. Vom Namensregister zum agilen Recherchewerkzeug. Überlegungen zur Geschichte und Wandel von Professorenkatalogen aus der Sicht des Catalogus Professorum Lipsiensis. In Professorinnen und Professoren gewinnen. Zur Geschichte des Berufungswesens an den Universitäten Mitteleuropas, Hrsg. Christian von Hesse und Rainer Christoph Schwinges, 441-470. Basel: Schwabe Verlag.

Musselin, Christine. 2005. European academic labor markets in transition. Higher Education 49(12): $135-154$.

Musselin, Christine. 2009. The market for academics. New York: Routledge.

Nielsen, Mathias W. 2016. Limits to meritocracy? Gender in academic recruitment and promotion processes. Science and Public Policy 43(3): 386-399.

Paletschek, Sylvia. 2012. Berufung und Geschlecht. Berufungswandel an bundesrepublikanischen Universitäten im 20. Jahrhundert. In Professorinnen und Professoren gewinnen. Zur Geschichte des Berufungswesens an den Universitäten Mitteleuropas, Hrsg. Christian von Hesse und Rainer Christoph Schwinges, 307-349. Basel: Schwabe.

Platt, Jennifer. 2015. Biographie in der Soziologiegeschichte. In Soziologiegeschichte: Wege und Ziele, Hrsg. Christian von Dayé und Stephan Moebius, 149-191. Berlin: Suhrkamp.

Plümper, Thomas, und Frank Schimmelpfennig. 2007. Wer wird Prof - und wann? Berufungsdeterminanten in der deutschen Politikwissenschaft. Politische Vierteljahresschrift 48(1): 97-117.

Prior, Lindsay. 2008. Repositioning documents in social research. Sociology 42(5): 821-836.

Prior, Lindsay. 2012. Using documents in research. Los Angeles: Sage.

Rebenich, Stefan. 2012. ,Geben Sie ihm eine gute Ermahnung mit auf den Weg und den Ordinarius. Berufungspolitik und Schulbildung in der Alten Geschichte. In Professorinnen und Professoren gewinnen. Zur Geschichte des Berufungswesens an den Universitäten Mitteleuropas, Hrsg. Christian von Hesse und Rainer Christoph Schwinges, 353-372. Basel: Schwabe.

Schmitt, Tassilo, Nina Arnhold, und Magnus Rüde. 2004. Berufungsverfahren im internationalen Vergleich. Gütersloh: CHE.

Smith, Dorothy E. 2007. The social construction of documentary reality. Sociological Inquiry 44(4): 257-268.

Stambolis, Barbara. 2012. Beruf und Berufung(en). Historiker des Jahrgangs 1943 im Rückblick auf ihr Leben mit der Geschichte. In Professorinnen und Professoren gewinnen. Zur Geschichte des Berufungswesens an den Universitäten Mitteleuropas, Hrsg. Christian von Hesse und Rainer Christoph Schwinges, 491-509. Basel: Schwabe.

Stark, Laura. 2011. Meetings by the minute(s). How documents create decisions for institutional review boards. In Social knowledge in the making, Hrsg. Charles von Camic, Neil Gross und Michèle Lamont, 233-255. Chicago/London: University of Chicago Press.

Trace, Ciaran B. 2002. What is recorded is never simply, what happened': Record keeping in modern organizational culture. Archival Science 2(1): 137-159.

Tsay, Angela, Michèle Lamont, Andrew Abbott, und Joshua Guetzkow. 2003. From character to intellect: Changing conceptions of merit in the social sciences and humanities, 19511971. Poetics 2003(31): 23-49.

van Langenhove, Luk, und Rom Harré. 1999. Positioning and the writing of science. In Positioning theory. Moral contexts of intentional action, Hrsg. Rom von Harré und Luk van Langenhove, 102-115. Oxford/Malden: Blackwell.

Weber, Wolfgang. 1984. Priester der Klio. Historisch-sozialwissenschaftliche Studien zur Herkunft und Karriere deutscher Historiker und zur Geschichte der Geschichtswissenschaft 1800-1970. Frankfurt a. M./Bern/New York: Peter Lang.

Wissenschaftsrat. 2005. Empfehlungen zur Ausgestaltung von Berufungsverfahren. Bonn: Wissenschaftsrat. 
Zimmerman, Don H., und Melvin Pollner. 1971. The everyday world as a phenomenon. In Understanding everyday life, Hrsg. Jack D. von Douglas, 80-103. London: Routledge/Kegan Paul.

Zimmermann, Karin. 2000. Spiele mit der Macht. Passfähigkeit und Geschlecht als Kriterien für Berufungen. Berlin: Edition Sigma. 